\title{
Evaluation of feline coronavirus viraemia in clinically healthy and ill cats with feline infectious peritonitis
}

\begin{abstract}
Feline Coronavirus (FCoV) comprises virulent and avirulent biotypes. While both biotypes can enter the bloodstream of a cat, only the virulent biotypes would replicate in monocytes and macrophages and develop a fatal disease known as Feline Infectious Peritonitis (FIP). In the present study, FCoV viraemia was evaluated in 50 cats consisting of 40 overtly healthy and 10 ill cats suspected of FIP. The blood samples were screened for FCoV genomic RNA by a RT-PCR assay and then followed by a duplex RT-PCR for detection of replicating viral mRNA. In the healthy cats, the virus and its replicating mRNA were detected in 67.5 and $15 \%$, respectively. The later finding suggested that the virus was replicating in a few cats with no clinical sign shown and indicated that FCoV viraemia do not necessarily lead to FIP. Probably the avirulent virus does multiply at low level in the blood or cat can harbor the virulent virus in an early stage of FIP without clinical signs yet. In FIP-suspected cases, all of the ill cats were positive for both FCoV and the replicating viral mRNA suggesting that FCoV could have replicated in blood and produced high amount of the virus and its components which were detectable by the both assays. The duplex RT-PCR assay which has been used to detect the replicating viral mRNA in blood was more specific than the general screening RT-PCR test for the diagnosis of FIP. The RT-PCR results however, should be interpreted in conjunction with other clinical symptoms.
\end{abstract}

Keyword: Feline coronavirus (FCoV); Feline infectious peritonitis (FIP); Cat blood; Viraemia; Symptom; Malaysia 